\title{
Evolution in student conceptual understanding of electricity and magnetism
}

\author{
Mary Jane Brundage and Chandralekha Singh \\ Department of Physics and Astronomy, University of Pittsburgh, Pittsburgh, PA, 15260
}

The Conceptual Survey of Electricity and Magnetism (CSEM) is a multiple-choice survey that contains a variety of electricity and magnetism concepts at the level of introductory physics used to help inform instructors of student mastery of those concepts. Prior studies suggest that many concepts on the survey are challenging for introductory physics students and the average student scores after traditional instruction are low. The research presented here compares the performance of students in introductory, upper-level, and graduate-level physics courses on five CSEM questions to understand the cross-sectional evolution of student understanding of these concepts from the introductory to upper-level to graduate-level. We discuss five CSEM questions that remain challenging for many upper-level and graduate students.

2021 PERC Proceedings edited by Bennett, Frank, and Vieyra; Peer-reviewed, doi.org/10.1119/perc.2021.pr.Brundage Published by the American Association of Physics Teachers under a Creative Commons Attribution 4.0 license. Further distribution must maintain the cover page and attribution to the article's authors. 


\section{INTRODUCTION AND GOAL}

One goal of an introductory electricity and magnetism course for physical science and engineering majors is for students to develop a robust conceptual understanding of the underlying physics [1-15]. This remains one of the goals in courses for physics majors as well as for physics graduate students as they take advanced physics courses even though their courses use complex mathematics to solve problems [16]. In these courses, students are often expected to make the mathphysics connection themselves to develop a good knowledge structure without much scaffolding support from instructors $[17,18]$. In particular, the focus of upper-level undergraduate and graduate courses is often on being able to solve complex quantitative problems with course assessment focusing exclusively on such problems, which students can do without a robust understanding of the underlying concepts.

In the context of electricity and magnetism (EM), students learn about the basic concepts including the Maxwell equations in integral form in the calculus-based introductory courses. However, in the upper-level undergraduate and graduate EM courses, students are taught to use differential forms of the Maxwell equations to solve complex problems often without any course assessment focusing on conceptual understanding. If students are not given grade incentives or provided support to make the appropriate math-physics connection, they may be able to solve problems in advanced courses using algorithmic approaches by pattern matching without developing a deep conceptual understanding [17, 18].

Many validated conceptual multiple-choice assessments have been developed to evaluate student understanding of basic concepts covered in introductory physics [19-26]. The research reported here compares the conceptual understanding of upper-level undergraduates and first year graduate students at a large research university with that of introductory students in calculus-based physics courses using a large national data on a validated EM survey suitable for introductory physics called The Conceptual Survey of Electricity and Magnetism or CSEM [20]. Our goal here is to investigate the extent to which advanced students have mastered these concepts compared to introductory students and what difficulties persist even for advanced students.

The CSEM has been used widely to investigate the conceptual understanding of students in introductory EM courses before and after instruction in relevant concepts. It is a 32question multiple-choice survey in which EM topics range from Coulomb's law to Faraday's law. The CSEM can be used to measure the effectiveness of evidence-based curricula and pedagogies. Past research using other surveys has shown that even in upper-level undergraduate classes, students have difficulty with many introductory and advanced level concepts [27, 28].

Here we use the CSEM to study the persistent difficulties of upper-level undergraduates and graduate students with EM concepts measured by the CSEM. In this paper, we discuss five questions from the CSEM that were challenging for undergraduates after instruction in upper-level EM. Our re- search questions are as follows:

RQ1: How difficult are these CSEM questions for the upper-level undergraduate and graduate students compared to the introductory students based upon their performance?

RQ2: What are the common difficulties of advanced students based upon the alternative choices they commonly selected? Are they the same or different from the alternative choices selected commonly by the introductory students?

\section{METHODOLOGY}

We use the CSEM to compare the EM conceptual knowledge of students in calculus-based introductory physics courses before and after instruction with students in a required upper-level physics undergraduate EM course before and after traditional instruction, and with graduate students enrolled in a teaching of physics course before their first-year physics graduate EM core course.

Introductory students and upper-level undergraduate students took the CSEM before (pre) and after (post) instruction in relevant concepts, whereas the graduate students only took it as a pretest. The introductory students took a calculusbased course in their first or second year. Data were collected over one semester in large-enrollment classes. Students took the CSEM both as a pre-test within the first week of classes and as post-test in the last few weeks. The upper-level EM course is required for physics majors and is usually taken by students in their second or third year. This course used Griffithâs Introduction to Electrodynamics as the textbook. Data for this course were collected over 3 different years and averaged together. Students took the CSEM both as a pre-test within the first week of classes and as post-test in the last few weeks. The graduate class consisted of first year physics graduate students in a Teaching of Physics class that prepared them for being teaching assistants. Data were collected from 4 different years and students took the CSEM as a pre-test, prior to their graduate EM instruction. All students used paper scantrons to bubble their responses for each question and had approximately 50 minutes, which was sufficient to answer all questions on CSEM. All three levels of students were from the same large research university.

The five questions from the CSEM to be discussed here are questions 13, 14, 21, 29, and 31 and they are shown below to give clarity to the underlying concepts. The answers for each question are bolded, italicized, and underlined.

13. Figure 1 shows a hollow conducting metal sphere which was initially given an evenly distributed positive (+) charge on its surface. Then a positive charge $+Q$ was brought up near the sphere as shown. What is the direction of the electric field at the center of the sphere after the positive charge $+Q$ is brought near the sphere?
(a) Left
(b) Right
(c) Up
(d) Down
(e) Zero field 


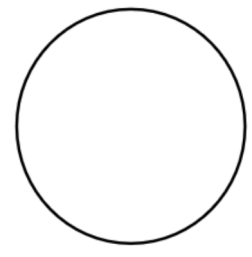

$$
+\mathrm{Q}
$$

FIG. 1: Figure for CSEM question 13.

14. Figure 2 shows an electric charge $q$ at the center of a hollow uncharged conducting metal sphere. Outside the sphere is a second charge Q. Both charges are positive. Choose the description below that describes the net electrical forces on each charge in this situation.

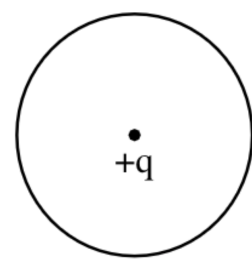

\section{$\dot{+Q}$}

FIG. 2: Figure for CSEM question 14.

(a) Both charges experience the same net force directed away from each other.

(b) No net force is experienced by either charge.

(c) There is no force on $\mathrm{Q}$ but a net force on $\mathrm{q}$.

(d) There is no force on $q$ but a net force on $Q$.

(e) Both charges experience a net force but they are different from each other.

21. What happens to a positive charge that is placed at rest in a uniform magnetic field? (A uniform field is one whose strength and direction are the same at all points.)

(a) It moves with a constant velocity since the force has a constant magnitude.

(b) It moves with a constant acceleration since the force has a constant magnitude.

(c) It moves in a circle at a constant speed since the force is always perpendicular to the velocity.

(d) It accelerates in a circle since the force is always perpendicular to the velocity.

\section{(e) It remains at rest since the force and the initial} velocity are zero.

29. The five separate figures (in Fig. 3) involve a cylindrical magnet and a tiny light bulb connected to the ends of a loop of copper wire. These figures are to be used in the following question. The plane of the wire loop is perpendicular to the reference axis. The states of motion of the magnet and of the loop of wire are indicated in the diagram. Speed will be represented by $\mathrm{v}$ and $\mathrm{CCW}$ represents counter clockwise. In which of the above figures will the light bulb be glowing?
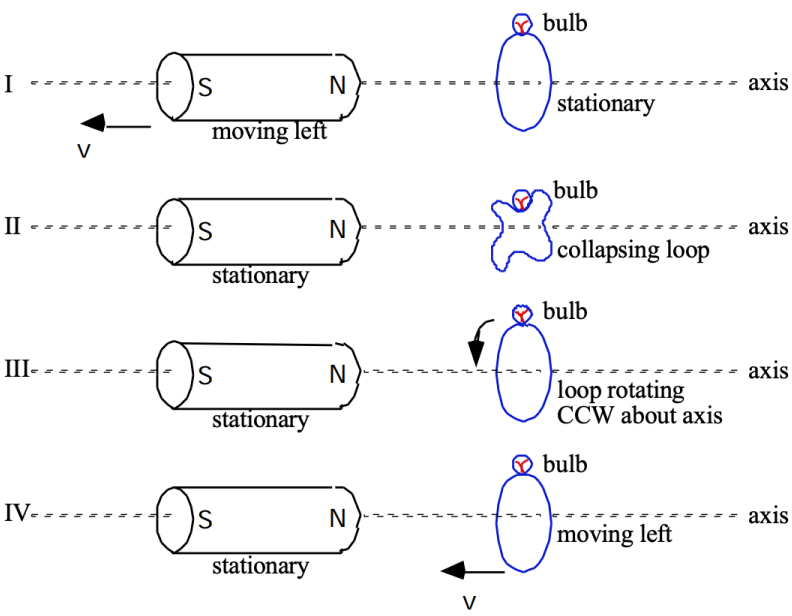

FIG. 3: Figure for CSEM question 29.
(a) I, III, IV
(b) I, IV
(c) I, II, IV
(d) IV
(e) None of these

31. A neutral bar (Fig. 4) is moving at a constant velocity v to the right through a region where there is a uniform magnetic field pointing out of the page. The magnetic field is produced by some large coils which are not shown on the diagram.

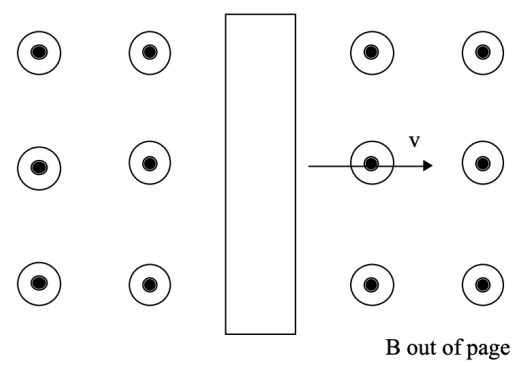

FIG. 4: Figure for CSEM question 31.

Which one of the following diagrams best describes the charge distribution on the surface of the metal bar?
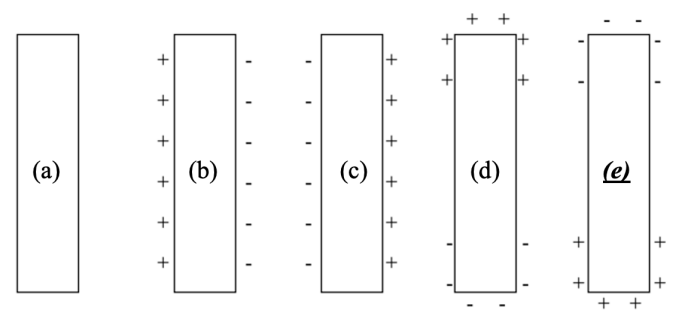

FIG. 5: Figure for CSEM question 31 answer choices. 


\section{RESULTS AND DISCUSSION}

There were 456 students who took the introductory level pre-test (Intro-pre), 401 students who took the introductory level post-test (Intro-post), 93 students who took the upperlevel pre-test (UL Pre), 85 students who took the upper-level post-test (UL Post), and there were 87 students in the graduate level class (Grad). We present data from all upperlevel students who took the pre/posttests because using only matched data for pre/posttest yields very similar outcomes. Table 1 shows the distribution of mean percentages for answer choices for the five CSEM questions with the correct answers bolded, italicized, and underlined. Percentages for a given question were calculated from students who did not leave that question blank (since some students skipped a few questions on the survey).

TABLE I. Distribution of mean percentages for answer choices for the five CSEM questions (Q) discussed here by the introductory (Intro), upper-level (UL), and graduate (Grad) students. Correct answers are bolded, italicized, and underlined. Showing all answer choices allows comparison of answer choices that were common.

\begin{tabular}{|c|c|c|c|c|c|c|}
\hline $\begin{array}{c}\text { CSEM } \\
\text { Q\# }\end{array}$ & & A & B & $\mathrm{C}$ & $\mathrm{D}$ & $\mathrm{E}$ \\
\hline \multirow{5}{*}{13} & Intro-Pre & $55 \%$ & $19 \%$ & $6 \%$ & $4 \%$ & $17 \%$ \\
\hline & Intro-Post & $26 \%$ & $17 \%$ & $2 \%$ & $2 \%$ & $\overline{54 \%}$ \\
\hline & UL Pre & $33 \%$ & $15 \%$ & $0 \%$ & $0 \%$ & $52 \%$ \\
\hline & UL Post & $19 \%$ & $17 \%$ & $0 \%$ & $2 \%$ & $\overline{62 \%}$ \\
\hline & Grad & $10 \%$ & $13 \%$ & $0 \%$ & $0 \%$ & $77 \%$ \\
\hline \multirow{5}{*}{14} & Intro-Pre & $55 \%$ & $9 \%$ & $5 \%$ & $15 \%$ & $\overline{16 \%}$ \\
\hline & Intro-Post & $34 \%$ & $14 \%$ & $5 \%$ & $38 \%$ & $9 \%$ \\
\hline & UL Pre & $46 \%$ & $9 \%$ & $3 \%$ & $31 \%$ & $12 \%$ \\
\hline & UL Post & $29 \%$ & $16 \%$ & $1 \%$ & $44 \%$ & $9 \%$ \\
\hline & Grad & $35 \%$ & $10 \%$ & $0 \%$ & $38 \%$ & $16 \%$ \\
\hline \multirow{5}{*}{21} & Intro-Pre & $20 \%$ & $34 \%$ & $22 \%$ & $\overline{12 \%}$ & $12 \%$ \\
\hline & Intro-Post & $14 \%$ & $30 \%$ & $27 \%$ & $13 \%$ & $\overline{17 \%}$ \\
\hline & UL Pre & $7 \%$ & $22 \%$ & $16 \%$ & $13 \%$ & $42 \%$ \\
\hline & UL Post & $4 \%$ & $13 \%$ & $16 \%$ & $11 \%$ & $57 \%$ \\
\hline & Grad & $2 \%$ & $11 \%$ & $15 \%$ & $2 \%$ & $\overline{69 \%}$ \\
\hline \multirow{5}{*}{29} & Intro-Pre & $31 \%$ & $25 \%$ & $20 \%$ & $20 \%$ & $5 \%$ \\
\hline & Intro-Post & $21 \%$ & $25 \%$ & $\underline{34 \%}$ & $19 \%$ & $2 \%$ \\
\hline & UL Pre & $16 \%$ & $28 \%$ & $37 \%$ & $18 \%$ & $0 \%$ \\
\hline & UL Post & $11 \%$ & $21 \%$ & $\overline{56 \%}$ & $12 \%$ & $0 \%$ \\
\hline & Grad & $3 \%$ & $25 \%$ & $\underline{61 \%}$ & $6 \%$ & $5 \%$ \\
\hline \multirow{5}{*}{31} & Intro-Pre & $8 \%$ & $27 \%$ & $39 \%$ & $17 \%$ & $7 \%$ \\
\hline & Intro-Post & $11 \%$ & $25 \%$ & $33 \%$ & $13 \%$ & $18 \%$ \\
\hline & UL Pre & $18 \%$ & $18 \%$ & $13 \%$ & $21 \%$ & $\underline{31 \%}$ \\
\hline & UL Post & $12 \%$ & $8 \%$ & $5 \%$ & $17 \%$ & $58 \%$ \\
\hline & Grad & $14 \%$ & $1 \%$ & $2 \%$ & $14 \%$ & $68 \%$ \\
\hline
\end{tabular}

Question 13 is the first of two questions that investigated student understanding of electric field and force on a charge inside of a conductor. The situation involves a charge distribution on the outside of a conducting sphere and a positive charge to the right of the sphere. In order to answer the question correctly, students must recognize that the conductor will rearrange the charges on the outside surface to create zero electric field inside it in equilibrium. Table I shows that at $54 \%$ of introductory students and $62 \%$ of upper-level undergraduates after instruction chose the correct response to question 13. At the graduate level, $77 \%$ of the students provided the correct response.

Question 14 builds on question 13 and evaluated student conceptual understanding of the electric force on a charge inside of a hollow conductor. In order to answer correctly, students must realize that in equilibrium, there is no electric field due to outside charges inside of a conductor. The outside charges induce charges on the surfaces of the conductor to make the net electric field zero due to outside charges. In particular, students must realize that since the net electric field from the outside sources at the point where charge $+q$ is located is zero, there would be no net force on $+q$. However, since charge $+Q$ is outside the conductor, it does experience the electric force due to $+\mathrm{q}$. Table I shows that this question was the most challenging of the five questions and less than $45 \%$ of any group of students provided the correct answer.

Contemplating student responses to questions 13 and 14, we infer that in the contexts provided, while students realized that there is no electric field inside a charged conductor, they did not realize that there would be no force on a charge inside a conductor due to the charge outside. For example, $77 \%$ of graduate students correctly answered question 13 about the electric field inside of a conductor, but only $38 \%$ of them answer question 14 correctly. The most common incorrect answer was choice (a), i.e., the net force on each charge is equal in magnitude and opposite in direction. Thus, students used Newtonâs 3rd law type reasoning to answer the question, which would have been correct if there was no conductor. It appears that even advanced students struggle to reason about how induced charges on the surface of a conductor shield the inside hollow region from charges outside.

Question 21 focuses on a charge at rest in a magnetic field. In order to answer the question correctly, students must recognize that in a uniform magnetic field, if a charge is initially at rest, it will continue to be at rest since there is no magnetic force on it. Table I shows that $17 \%$ of introductory students and $57 \%$ of upper-level students provided the correct response after instruction in relevant concepts, and 69\% of the graduate students answered this question correctly before instruction in graduate EM. The most common incorrect choice among upper-level undergraduates and graduate students was (c), i.e., the particle would move in a circle with a constant speed since the force is always perpendicular to the velocity. This alternative conception shows that these students did not realize that a charged particle moves in a circle with a constant speed in a uniform magnetic field only if the initial velocity of the particle is perpendicular to the field. In other words, students must recognize that a charged particle experiences a non-zero magnetic force only when it is moving at an angle other than $0^{\circ}$ or $180^{\circ}$ to the magnetic field.

Question 29 shows different situations in which students must determine if a lightbulb will light up due to induced current or not. In particular, students must recognize that the lightbulb in the loop of wire will light up when a current flows through the wire (in this case, an induced current flows due to an induced emf created by the changing magnetic 
flux through the loop). Students should realize that the flux through the loop is a measure of the number of magnetic field lines passing through its surface area and a collapsing loop would mean a decrease in area and a change in flux. Students must also recognize that along the axis of a bar magnet, the strength of the magnetic field is strongest near the poles and weaker far away so that when the distance between the loop of wire and the bar magnet decreases, the strength of the magnetic field passing through the loop would increase and vice versa. The correct answer is choice (c). Only $34 \%$ of the introductory students and $56 \%$ of upper-level students after instruction answered the question correctly. Also, only $61 \%$ of graduate students selected the correct response. The most common incorrect choice is (b), which shows situations I and IV, leaving out II, in which the loop is collapsing. Table 1 shows that $25 \%$ of introductory, $21 \%$ of upper-level students after instruction, and $25 \%$ of graduate students did not realize that the light bulb would light up in case II in which the loop collapses. This suggests that these students did not realize that collapsing the loop in this case will lead to a decrease in flux through the loop and hence cause an induced current.

Question 31 involves the concept of motional emf associated with a metal bar moving in a uniform magnetic field and asked students to choose the correct charge distribution on the metal bar among the five choices. In order to answer the question correctly, students must recognize that since the metal bar has free electrons, the right-hand rule can be used to find the direction of the force on the electrons when they move to the right (in the moving bar shown in Fig. 4) and the magnetic field points out of the page. In particular, they must realize that the downward force would act on the positive charges, and an upward force on the electrons. Thus, the emf induced would have positive charges accumulating on the bottom and the negative charges accumulating on the top of the metal bar as shown in choice (e) in Fig. 5. Only $18 \%$ of introductory students, $58 \%$ of upper-level students after instruction, and $68 \%$ of the graduate students selected the correct response. The most common incorrect answer for graduate students is tied between options (a) and (d), with $14 \%$ of students selecting each choice at the graduate level. The incorrect choice (d) shown the opposite charge separation to the correct answer and may signify difficulty with using the right-hand rule correctly in this context. The incorrect option (a) shows an uncharged bar, which signifies that the students who selected this answer thought that a neutral metal bar moving in a uniform magnetic field would not have any charge separation. Although this situation of a motional emf produced in a metal bar moving in a uniform magnetic field is routinely discussed especially in introductory physics courses, it is possible that at least some of these students who selected option (a) thought that an emf would only be produced if this metal bar was part of a complete circuit. In other words, they may have confused the fact that in the situation in question 31 there would be an induced emf even though an induced current would require a complete circuit.

\section{SUMMARY AND FUTURE DIRECTIONS}

We investigated the difficulty on five CSEM questions for the introductory and upper-level undergraduates and graduate students at a large research university in the US and compared their performance. We also investigated the common difficulties of advanced students based upon the alternative choices they commonly selected and analyzed whether they were the same or different from the alternative choices selected commonly by the introductory students. We find that many upperlevel physics undergraduates after instruction in relevant concepts in their upper-level EM course and first-year graduate students before instruction in graduate EM struggled with the underlying concepts in these questions that are designed to be at the level of EM covered in introductory courses. Furthermore, analysis of upper-level undergraduate students' performance on all CSEM questions after instruction (to be discussed elsewhere in detail) shows that if introductory students did not reach $50 \%$ correct on a question after instruction, less than two thirds of the upper-level students provided the correct response for that question after traditional instruction.

These findings make us contemplate whether the manner in which the traditional upper-level undergraduate EM course is being taught is achieving its goals or whether it is a missed learning opportunity. In particular, upper-level students may become proficient in solving complex problems algorithmically but without an effort to also develop a deep conceptual understanding of the underlying content simultaneously, they are not likely to retain much of what is taught.

We have begun to interview upper-level course instructors and shared these data with them to understand their perspectives on these issues. For example, we asked some instructors who have taught these upper-level courses what these findings mean to them and whether they would make more effort to integrate conceptual and quantitative aspects of physics both in their lectures and on their course assessments since assessment drives learning. Preliminary findings suggest that some instructors feel that they already discuss physics concepts throughout in these advanced physics courses, but they do not assess students on their conceptual understanding since they expect them to have learned the underlying concepts in their introductory courses. Interviews suggest that some instructors feel that the goal of the advanced physics courses is to help students learn to use sophisticated mathematics to do complex problems and it is the students' responsibility to reflect upon how it connects with the underlying concepts. Some acknowledged that they expect that students would recognize that it is important for them to build on their prior knowledge of relevant concepts in order to retain what they have learned in their advanced courses. They noted that while they did not have the time to contemplate how to integrate conceptual and quantitative aspects of the content in their teaching and assessment, if others had developed materials, they would be willing to try them in their classes. The persistent difficulties of advanced students such as those discussed here should be taken into account to improve their learning. 
[1] L. McDermott, Millikan Lecture 1990: What we teach and what is learned-Closing the gap, Am. J. Phys. 59, 301 (1991).

[2] L. McDermott and P. Shaffer, Research as a guide for curriculum development: An example from introductory electricity. Part I: Investigation of student understanding, Am. J. Phys. 60, 994 (1992).

[3] P. Shaffer and L. McDermott, Research as a guide for curriculum development: An example from introductory electricity. Part II: Design of instructional strategies, Am. J. Phys. 60, 1003 (1992).

[4] L. McDermott, P. Shaffer, and the PEG, Tutorials in Introductory Physics, 2nd ed. (Prentice Hall, 2002).

[5] C. Singh, Impact of peer interaction on conceptual test performance, Am. J. Phys. 73, 446 (2005).

[6] Z. Isvan and C. Singh, Improving student understanding of Coulomb's law and Gauss's Law, AIP Conf. Proc. 883, 181 (2007).

[7] J. Li and C. Singh, Developing a magnetism conceptual survey and assessing gender differences in student understanding of magnetism, AIP Conf. Proc. 1413, 43 (2012).

[8] J. Li and C. Singh, Students' difficulties with equations involving circuit elements, AIP Conf. Proc. 1413, 243 (2012).

[9] J. Li and C. Singh, Students' common difficulties and approaches while solving conceptual problems with non-identical light bulbs in series and parallel, Eur. J. Phys 37, 065708 (2016).

[10] A. Maries and C. Singh, Do students benefit from drawing productive diagrams themselves while solving introductory physics problems? the case of two electrostatics problems, Eur. J. Phys 39, 015703 (2017).

[11] J. Li and C. Singh, Investigating and improving introductory physics students' understanding of electric field and superposition principle, Eur. J. Phys 38, 055702 (2017).

[12] J. Li and C. Singh, Investigating and improving introductory physics students' understanding of electric field and the superposition principle: The case of a continuous charge distribution, Phys. Rev. Phys, Educ. Res 15, 10116 (2019).

[13] J. Li and C. Singh, Investigating and improving student understanding of symmetry and Gauss's law, Eur. J. Phys. 39, 015702 (2018).

[14] A. Maries and C. Singh, Case of two electrostatics problems: Can providing a diagram adversely impact introductory physics students' problem solving performance?, Phys. Rev. Phys. Educ. Res. 14, 010114 (2018).
[15] N. Karim, A. Maries, and C. Singh, Exploring one aspect of pedagogical content knowledge of teaching assistants using the Conceptual Survey of Electricity and Magnetism, Phys. Rev. Phys, Educ. Res 14, 10117 (2018).

[16] C. Singh and A. Maries, Core graduate courses: A missed learning opportunity?, AIP Conf. Proc. 1513, 382 (2013).

[17] A. Maries, S. Lin, and C. Singh, Challenges in designing appropriate scaffolding to improve students' representational consistency: The case of a Gauss's law problem, Phys. Rev. Phys, Educ. Res 13, 1 (2017).

[18] E. Redish, Using Math in Physics: Overview, Phys. Teach 59, 314 (2021).

[19] D. Hestenes, M. Wells, and G. Swackhamer, Force concept inventory, Phys. Teach 30, 141 (1992).

[20] D. Maloney, T. O'Kuma, C. Hieggelke, and A. Van Heuvelen, Surveying students' conceptual knowledge of electricity and magnetism, Am. J. Phys. 69, S12 (2001).

[21] C. Singh and D. Rosengrant, Multiple-choice test of energy and momentum concepts, Am. J. Phys. 71, 607 (2003).

[22] L. Rimoldini and C. Singh, Student understanding of rotational and rolling motion concepts, Phys. Rev. ST Phys. Educ. Res. 1, 010102 (2005).

[23] C. Singh, Student understanding of symmetry and Gauss's law of electricity, Am. J. Phys. 74, 923 (2006).

[24] L. Ding, R. Chabay, B. Sherwood, and R. Beichner, Evaluating an electricity and magnetism assessment tool: Brief electricity and magnetism assessment, Phys. Rev. Spec. Top. - Phys. Educ. Res. 2, 010105 (2006).

[25] J. Li and C. Singh, Developing and validating a conceptual survey to assess introductory physics students' understanding of magnetism, Eur. J. Phys. 38, 025702 (2017).

[26] B. Brown and C. Singh, Development and validation of a conceptual survey instrument to evaluate students' understanding of thermodynamics, Phys. Rev. Phys. Educ. Res. 17, 010104 (2021).

[27] S. Pollock, Longitudinal study of student conceptual understanding in electricity and magnetism, Phys. Rev. Spec. Top. - Phys. Educ. Res. 5, 020110 (2009).

[28] B. Wilcox, M. Caballero, C. Baily, H. Sadaghiani, S. Chasteen, Q. Ryan, and S. Pollock, Development and uses of upperdivision conceptual assessments, Phys. Rev. Spec. Top. - Phys. Educ. Res. 11, 1 (2015). 